\title{
How many tigers Panthera tigris are there in Huai Kha Khaeng Wildlife Sanctuary, Thailand? An estimate using photographic capture-recapture sampling
}

\author{
Saksit Simcharoen, Anak Pattanavibool, K. Ullas Karanth, James D. Nichols and N. Samba Kumar
}

\begin{abstract}
We used capture-recapture analyses to estimate the density of a tiger Panthera tigris population in the tropical forests of Huai Kha Khaeng Wildlife Sanctuary, Thailand, from photographic capture histories of 15 distinct individuals. The closure test results $(z=0.39, \mathrm{P}=0.65)$ provided some evidence in support of the demographic closure assumption. Fit of eight plausible closed models to the data indicated more support for model $\mathrm{M}_{\mathrm{h}}$, which incorporates individual heterogeneity in capture probabilities. This model generated an average capture probability $\hat{p}=0.42$ and an abundance estimate of $\widehat{N}(\widehat{S E}[\widehat{N}])=19$ (9.65) tigers. The sampled area of $\widehat{A}(W)(\widehat{S E}[\widehat{A}(W)])=$ $477.2(58.24) \mathrm{km}^{2}$ yielded a density estimate of $\widehat{D}(\widehat{S E}[\widehat{D}])=$ 3.98 (0.51) tigers per $100 \mathrm{~km}^{2}$. Huai Kha Khaeng Wildlife Sanctuary could therefore hold 113 tigers and the entire
\end{abstract}

Western Forest Complex c. 720 tigers. Although based on field protocols that constrained us to use sub-optimal analyses, this estimated tiger density is comparable to tiger densities in Indian reserves that support moderate prey abundances. However, tiger densities in wellprotected Indian reserves with high prey abundances are three times higher. If given adequate protection we believe that the Western Forest Complex of Thailand could potentially harbour $>2,000$ wild tigers, highlighting its importance for global tiger conservation. The monitoring approaches we recommend here would be useful for managing this tiger population.

Keywords Camera traps, capture-recapture models, Panthera tigris, population estimation, Thailand, tiger.

\section{Introduction}

The tiger Panthera tigris is categorized as Endangered on the IUCN Red List (IUCN, 2006) and during the past 3 decades substantial efforts have been invested in tiger conservation by governments and non-governmental agencies. However, these efforts are constrained by a lack of reliable data on the distribution as well as densities of wild tiger populations. Furthermore, dissemination of putative 'tiger numbers' (Jackson, 1993), often based on guesswork or demonstrably faulty methods (Karanth, 1987, 1988; Karanth et al., 2003) masks a real scarcity of reliable data. Therefore, there is an urgent need

Saksit Simcharoen Wildlife Research Division, Department of National Park, Plant, and Wildlife Conservation, Paholyotin Road, Chatuchak, Bangkok 10900, Thailand.

Anak Pattanavibool Wildlife Conservation Society -Thailand Program, P.O.Box 170, Laksi, Bangkok 10210, Thailand.

K. Ullas Karanth (Corresponding author) and N. Samba Kumar Wildlife Conservation Society-India Program, Centre for Wildlife Studies, 26-2, Aga Abbas Ali Road (Apt: 430), Bangalore, Karnataka-560 042, India. E-mail ukaranth@wcs.org

James D. Nichols US Geological Survey, Patuxent Wildlife Research Center, Laurel, Maryland 20708, USA.

Received 1 February 2006. Revision requested 25 September 2006. Accepted 20 December 2006. to obtain reliable estimates of tiger densities at a large number of sites across the 1.2 million $\mathrm{km}^{2}$ geographic range of the species (Seidensticker et al., 1999).

Thailand is a key tiger range state, with $25 \%$ of its land area under forest cover, $16 \%$ of it being managed under wildlife and national park protection legislation (Pattanavibool \& Dearden, 2002). In addition, increasing societal wealth and an official commitment to sciencebased tiger conservation (Tunhikorn et al., 2004) make Thailand a critical region for tiger conservation. Consequently, attempts have been made to map accurately the distribution of tiger populations in Thailand from field surveys (Rabinowitz, 1993, 1999; Smith et al., 1999; Tunhikorn et al., 2004; WEFCOM, 2004). However, to use such maps for managing wild tiger populations there is an additional need to estimate densities and sizes of individual tiger populations at specific sites. This critical need has been enunciated in Thailand's national action plan for tigers (Tunhikorn et al., 2004). The national plan also identifies the $18,000 \mathrm{~km}^{2}$ Western Forest Complex, which contains 17 protected areas, including Huai Kha Khaeng Wildlife Sanctuary, as the most important tiger conservation area in the country.

Although reliable estimation of tiger abundance is difficult because of their elusive behaviour and naturally low densities, recent development of automated camera traps and their application within a formal framework 
of capture-recapture population sampling (see Karanth et al., 2004b, for a review) have enabled investigators to obtain rigorous density estimates in India (Karanth \& Nichols, 1998; Karanth et al., 2004a,c), Nepal (Wegge et al., 2004), Malaysia (Kawanishi \& Sunquist, 2004) and Indonesia (O'Brien et al., 2003). Unlike previous tiger monitoring approaches based on footprint total counts (Panwar, 1980), radio-telemetry (Sunquist, 1981; Smith, 1993) or raw photographic trapping rates (Carbone et al., 2001), capture-recapture methods can effectively deal with the typical inability of surveys to detect all individual tigers present in an area (i.e. detection probability P <1; Williams et al., 2002). Photographic capturerecapture sample surveys of tigers conducted in habitats ranging from evergreen, semi-deciduous and deciduous forests to alluvial grasslands (O'Brien et al., 2003; Karanth et al., 2004a; Kawanishi \& Sunquist, 2004; Wegge et al., 2004) show that reliable estimates can be generated at relatively low densities of 2-3 tigers per $100 \mathrm{~km}^{2}$, although their variances tend to be large because of the small number of traps typically deployed in such studies. A recent study (Karanth et al., 2006) that integrated photo-capture data across space and time employing the Robust Design (Pollock et al., 1990; Lebreton et al., 1992; Kendall et al., 1997; Williams et al., 2002) demonstrated the power of capture-recapture analyses to detect changes in the temporal dynamics of a tiger population.

However, prior to this study, there has not been an estimate of tiger abundance in Thailand based on capture-recapture analyses. Here we present the results of a post hoc capture-recapture analysis of camera trap survey data collected in Huai Kha Khaeng Wildlife Sanctuary during 2004-2005. The objectives of our analysis were to: (1) Assess the potential for employing camera trap surveys in the semi-deciduous forests that form a large proportion of tiger habitat in Thailand (Tunhikorn et al., 2004). (2) Analyse the tiger photocapture data in a formal capture-recapture sampling framework (Otis et al., 1978; White et al., 1982; Williams et al., 2002) to generate estimates of capture probability, population size, effectively sampled area and tiger density based on survey protocols developed in India (Karanth et al., 2002; Nichols \& Karanth, 2002). (3) Assess whether tiger densities in Huai Kha Khaeng are comparable to densities recorded in ecologically similar semi-deciduous forest sites in India (Karanth et al., 2004c). (4) Examine the general implications of our results for understanding tiger ecology and monitoring wild tiger populations in Thailand.

\section{Study area}

This study was carried out in the forests around Khao Nang Rum research station within the $2,780 \mathrm{~km}^{2}$ Huai
Kha Khaeng Wildlife Sanctuary (Fig. 1). The area is rugged and hilly over altitudes of 200-1,600 m, has an annual temperature range of $10-35^{\circ} \mathrm{C}$ and annual precipitation of $c$. $1,500 \mathrm{~mm}$. It supports four vegetation types: dry deciduous dipterocarp forests, mixed deciduous forest, dry evergreen forest, and hill evergreen forest, depending on rainfall patterns and edaphic factors (Srikosamatara, 1993; Tunhikorn et al., 2004; WEFCOM, 2004). From earlier food habit studies in the area (Petdee, 2000), principal prey species of tigers are wild pig Sus scrofa, sambar Cerous unicolor, common muntjac Muntiacus muntjac, banteng Bos javanicus and gaur Bos frontalis. Other potential tiger prey include wild buffalo Bubalus bubalis and Malayan tapir Tapirus indicus.

\section{Methods}

\section{Field methods}

The original goal was to document the presence of tigers and other mammals in the area using camera-trap techniques. Therefore, trapping was done on an ad hoc basis without employing recommended survey protocols (Karanth et al., 2002; Nichols \& Karanth, 2002). Twelve Trailmaster (Goodson \& Associates, Lenexa, USA) and 10 CamTrakker (CamTrakker, Georgia, USA) units were deployed to cover a $211 \mathrm{~km}^{2}$ area using 103 trap locations (Fig. 1).

Trapping was carried out from 9 February 2004 to 1 February 2005 using 14 clusters of trap locations. These clusters are analogous to trapping blocks (Nichols \& Karanth, 2002), with each block consisting of $c$. seven trap locations. The sampling effort varied among blocks: eight locations were trapped for $>20$ days, 49 locations for 16-19 days, 12 locations for c. 15 days and the remaining 34 locations were trapped for $<15$ days. On average there were $c .15$ trap-days at each location, and this trapping effort was uniform across the study area. The moving of traps among blocks did not follow a strict pre-designed sequence and was driven by logistics as well as opportunities for setting traps at tiger kill sites. However, in combination, data from all these blocks covered the area evenly (Fig. 1).

Of particular concern for the analysis was the long survey duration of 12 months, resulting in the possibility of the sampled tiger population being demographically open (Otis et al., 1978). Given the high turnover of individuals in tiger populations (Karanth et al., 2006), such lack of closure could bias estimates of population size. However, the following aspects of the survey encouraged us to attempt a post-hoc statistical analysis of these data under a formal capture-recapture sampling framework: (1) There were two opposing cameras at each trap location, at a distance of $c$. 3-5 $\mathrm{m}$ from the 


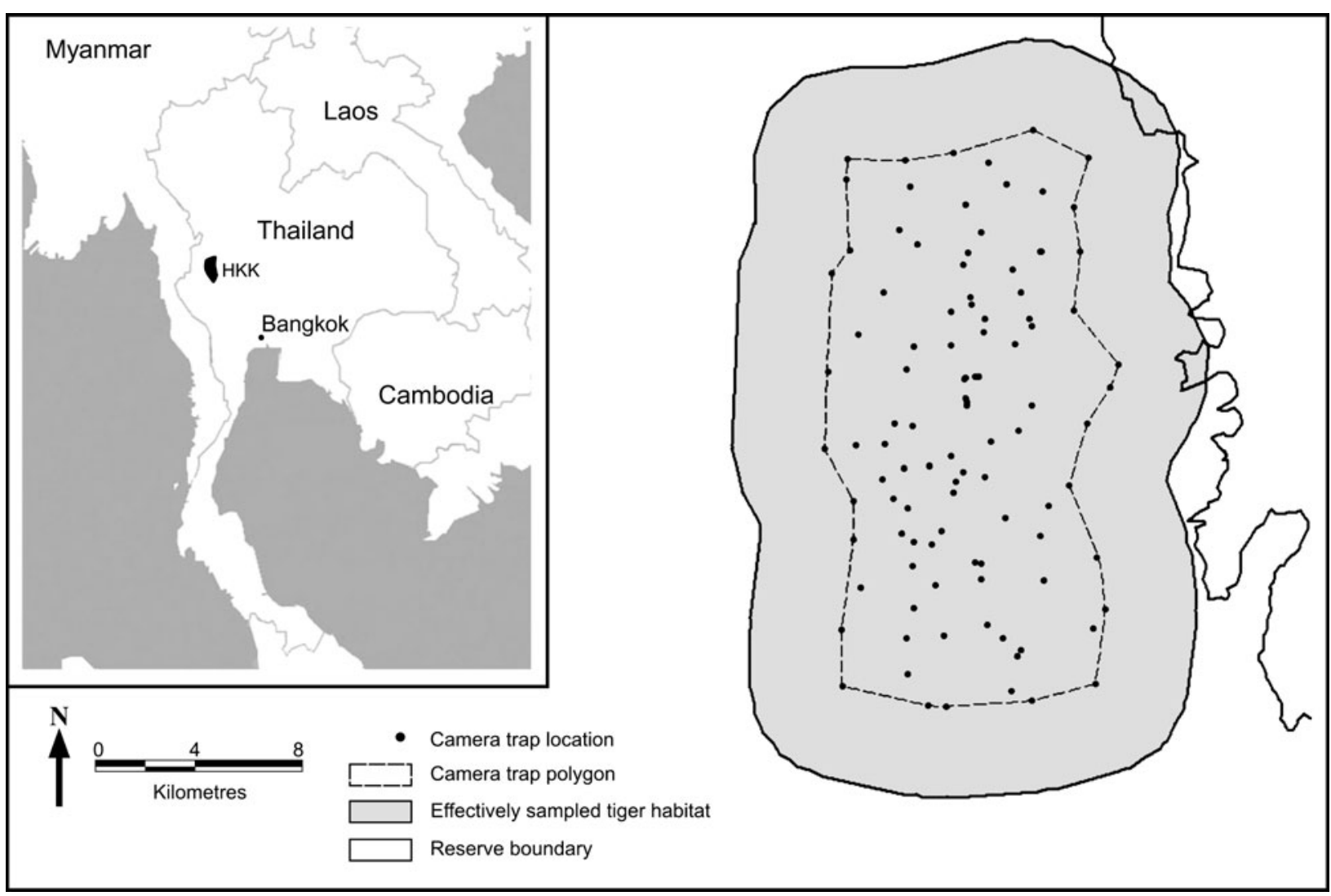

Fig. 1 The Khao Nang Rum camera-trap survey area in Huai Kha Khaeng Wildlife Sanctuary. Inset shows the Sanctuary's location (HKK) in Thailand.

anticipated path of moving tigers at c. $45 \mathrm{~cm}$ height, which obtained good photographs of both flanks, enabling unambiguous identification of individuals. (2) The camera trap locations were selected based on signs of past tiger activity to maximize capture probabilities, resulting in a relatively large number $(n=17)$ of individual tigers being photo-captured. (3) The maximum spacing between any two trap locations was $<2.3 \mathrm{~km}$, thus ensuring that there were no holes in the sampled area and that every tiger in the sampled population had a non-zero probability of being photo-captured during each sampling occasion.

\section{Analysis}

Given the potential for lack of demographic closure (Karanth et al., 2002; Williams et al., 2002) in the data we would have preferred to use open model analyses (Karanth et al., 2006). However, the lack of simultaneous natural temporal coverage of the entire survey area (because we had to construct our sampling occasions as described above) precluded this option. Therefore, we constructed closed model capture histories following survey design 4 of Nichols \& Karanth (2002), ensuring that capture data from well spaced locations were included in every sampling occasion. We constructed five sampling occasions based on the calendar dates on which each location was trapped (Otis et al., 1978; Karanth \& Nichols, 1998). Because of low capture rates, tiger photo-capture data from three successive calendar dates at each trapping location were combined before being assigned to a specific sampling occasion. We thus ensured that equal trapping effort was expended and the entire area was sampled during each of the sampling occasions.

The individual tiger capture histories in the standard X-matrix format (Otis et al., 1978; White et al., 1982) were analysed using models developed for closed populations (Otis et al., 1978; White et al., 1982) implemented in the software CAPTURE (Rexstad \& Burnham, 1991). We tested the population closure assumption against our data. The closure test (Otis et al., 1978; White et al., 1982) implemented in CAPTURE is based on the number of sample periods separating the times of first and last capture for each animal caught at least twice. If animals are entering and/or leaving the sampled population during the survey period, the time between first and last captures should be shorter on average than if all animals were present during the entire survey period. 
The capture-recapture models implemented in CAPTURE consider potential effects of behavioural response of tigers to camera trapping (e.g. trap-avoidance: model $\mathrm{M}_{\mathrm{b}}$ ), time-specific variation (e.g. weather changes over the 3-day sampling occasions: model $\mathrm{M}_{\mathrm{t}}$ ), and heterogeneity among individual animals (e.g. caused by factors such as territorial status or trap access: model $\mathrm{M}_{\mathrm{h}}$ ), as well as more complex models such as $\mathrm{M}_{\mathrm{bh}}, \mathrm{M}_{\mathrm{th}}, \mathrm{M}_{\mathrm{tb}}$ and $\mathrm{M}_{\mathrm{tbh}}$ that incorporate occurrence of the effects of heterogeneity, trap response and time in different combinations.

We fitted the null model $\mathrm{M}_{0}$ and each of the above seven models to our data using CAPTURE (Rexstad \& Burnham, 1991) and examined results of goodness-of-fit and betweenmodel tests, and the overall discriminant function, to guide the selection of an appropriate model for the data. The selected model was then used for estimating capture probabilities $\widehat{p}$ and abundance $\widehat{N}$. We estimated the effectively sampled area using an approach evaluated by Wilson \& Anderson (1985), and computed tiger densities by dividing the population size by the sampled area. This computational approach is fully described elsewhere (Karanth \& Nichols, 1998; Nichols \& Karanth, 2002).

\section{Results}

\section{Photographic captures of tigers}

In a total sampling effort of 1,509 trap-days we obtained 124 tiger photographs (59 right flanks, 57 left flanks, four frontal, four rear) of 15 individual tigers judged to be $>12$ months of age (10 females, four males, one of unknown sex). Individual tigers could be clearly identified from stripe patterns (Karanth et al., 2002) and were given unique identification numbers (HKT-101-HKT-117). We obtained both left and right profile photos for 12 individuals, and three more animals were identified from only left profiles. Capture data for two cubs were excluded from the analysis.

The capture histories generated from the field survey (Table 1) show that the number of individuals caught was small $\left(\mathrm{M}_{\mathrm{t}+1}=15\right)$, as expected in a low to medium density tiger population (Karanth et al., 2004c). Four animals were caught in all five sampling occasions, one was caught in four occasions, two animals were caught thrice, two others twice and six individual tigers were caught only once. We expected this low recapture rate for several individuals to induce substantial uncertainty in our estimates.

\section{Estimates of effectively sampled area}

The polygon formed by the outer-most camera traps (Fig. 1) was $211 \mathrm{~km}^{2}$. For the 10 individual tigers that were caught more than once, the maximum distance
Table 1 Capture histories of tigers photo-trapped in Huai Kha Khaeng Wildlife Sanctuary, Thailand, during 2004-2005.

\begin{tabular}{lllllll} 
& \multicolumn{3}{l}{ Sampling occasion } & \\
\cline { 2 - 5 } Identification no. & 1 & 2 & 3 & 4 & 5 & \multirow{2}{*}{ Age/sex } \\
\hline HKT-101 & 1 & 1 & 1 & 1 & 1 & $\mathrm{~F}$ \\
HKT-102 & 1 & 0 & 1 & 1 & 1 & $\mathrm{~F}$ \\
HKT-103 & 1 & 0 & 1 & 0 & 1 & $\mathrm{~F}$ \\
HKT-104 & 1 & 1 & 1 & 1 & 1 & $\mathrm{~F}$ \\
HKT-105 & 1 & 1 & 1 & 1 & 1 & $\mathrm{~F}$ \\
HKT-106 & 1 & 1 & 1 & 1 & 1 & $\mathrm{M}$ \\
HKT-107 & 0 & 0 & 0 & 1 & 1 & $\mathrm{M}$ \\
HKT-108 & 0 & 1 & 0 & 1 & 1 & $\mathrm{~F}$ \\
HKT-109 & 1 & 0 & 0 & 0 & 0 & $\mathrm{~F}$ \\
HKT-110 & 0 & 0 & 1 & 0 & 0 & $\mathrm{M}$ \\
HKT-111 & 0 & 0 & 0 & 0 & 1 & $\mathrm{~F}$ \\
HKT-112 & 0 & 1 & 0 & 0 & 0 & $\mathrm{U}$ \\
HKT-113 & 0 & 0 & 1 & 0 & 0 & $\mathrm{~F}$ \\
HKT-115 & 1 & 1 & 0 & 0 & 0 & $\mathrm{C}$ \\
HKT-116 & 0 & 1 & 0 & 0 & 0 & $\mathrm{C}$ \\
HKT-114 & 0 & 0 & 0 & 0 & 1 & $\mathrm{M}$ \\
HKT-117 & 1 & 0 & 1 & 0 & 0 & $\mathrm{~F}$ \\
\hline
\end{tabular}

${ }^{*} \mathrm{~F}$, female $>12$ months; $\mathrm{M}$, male $>12$ months; $\mathrm{U}$, unknown sex $>12$ months; $\mathrm{C}$, cubs $<12$ months (not included in the capture-recapture analysis).

between photo-captures was $0.90-16.05 \mathrm{~km}$, with a mean value of $7.11 \mathrm{~km}$. Using the approach described more fully elsewhere (Karanth \& Nichols, 1998; Nichols \& Karanth, 2002), we estimated the effectively sampled area as $\widehat{A}(W)(\widehat{S E}[\widehat{A}(W)])=477.2(58.24) \mathrm{km}^{2}$.

\section{Tests for population closure and model selection}

The statistical test for population closure implemented in CAPTURE (Rexstad \& Burnham, 1991) was consistent with the assumption that our tiger population was closed during the survey period $(z=0.39, \mathrm{P}=0.65)$. Because of the long (12 months) survey period, we would have liked to consider open models as well but the ad hoc field sampling design precluded this possibility. We assumed that our data supported the closure assumption, albeit not strongly.

The test for presence of individual heterogeneity in capture probabilities showed that the null model $\mathrm{M}_{0}$ was rejected in favour of the model incorporating heterogeneity $\mathrm{M}_{\mathrm{h}}\left(\chi^{2}=10.07, \mathrm{df}=1, \mathrm{P}<0.002\right)$. The goodness-of-fit test results for models $\mathrm{M}_{\mathrm{h}}$ and $\mathrm{M}_{\mathrm{b}}$ (incorporating trapresponse behaviour) provided no evidence of lack of fit $\left(\chi^{2}=3.85, \mathrm{df}=4, \mathrm{P}=0.43\right.$ and $\chi^{2}=2.57, \mathrm{df}=4$, $\mathrm{P}=0.64$, respectively). The tests also did not reject the null model $\mathrm{M}_{0}$ in favour of alternative models $\mathrm{M}_{\mathrm{b}}\left(\chi^{2}=\right.$ $0.77, \mathrm{df}=1, \mathrm{P}=0.38$ ) or $\mathrm{M}_{\mathrm{t}}$ (time-specific variation in capture probabilities; $\chi^{2}=2.86$, df $=4, \mathrm{P}=0.58$ ). Model $\mathrm{M}_{\mathrm{bh}}$, which accommodates heterogeneity as well as 
trap response was not favoured over the more parsimonious $\mathrm{M}_{\mathrm{h}}$ model $\left(\chi^{2}=0.67, \mathrm{df}=2, \mathrm{P}=0.72\right)$.

The overall discriminant function model selection algorithm in CAPTURE (Rexstad \& Burnham, 1991) scored the competing models as: $\mathrm{M}_{0}=0.88, \mathrm{M}_{\mathrm{h}}=1.00$, $\mathrm{M}_{\mathrm{b}}=0.38, \mathrm{M}_{\mathrm{bh}}=0.57, \mathrm{M}_{\mathrm{t}}=0.0, \mathrm{M}_{\mathrm{th}}=0.41, \mathrm{M}_{\mathrm{tb}}=$ $0.37, \mathrm{M}_{\mathrm{tbh}}=0.64$. The higher scoring model $\mathrm{M}_{\mathrm{h}}$ is more likely to have generated the observed capture history data in comparison to lower scoring models. This choice of model $\mathrm{M}_{\mathrm{h}}$ in statistical tests reported above is consistent with past results (Karanth et al., 2004c) as well as aspects of tiger biology. Resident breeding tigers maintain home ranges that overlap between the sexes. Additionally, some individuals in the population are nonbreeding 'floaters', which may not have stable home ranges (Sunquist, 1981; Smith, 1993; Karanth \& Sunquist, 2000). These space use patterns, as well as location of our camera traps in relation to home ranges of individuals, were likely to induce differences in capture probabilities among individual tigers.

\section{Estimates of capture probability, tiger population size and density}

The tiger capture histories (Table 1) were used to generate parameter estimates under model $\mathrm{M}_{\mathrm{h}}$ using the jackknife estimator (Burnham \& Overton, 1978; Otis et al., 1978) implemented in CAPTURE, which performed well in earlier photographic capture studies of tigers (Karanth \& Nichols, 1998; Karanth et al., 2004c). The estimated average capture probability per sampling occasion $(\widehat{p})$ was 0.42 . The total population size estimate $(\widehat{N})$ was 19 tigers with a standard error $(\widehat{S E}[\widehat{N}])$ of 3.9 tigers. Based on the sampled area $\widehat{A}(W)(\widehat{S E}[\widehat{A}(W)])=$ $477.2(58.24) \mathrm{km}^{2}$, the estimated density of tigers in the area was $\widehat{D}(\widehat{S E}[\widehat{D}])=3.98(0.51)$ tigers per $100 \mathrm{~km}^{2}$. These estimates exclude cubs $<12$ months in age, which generally comprise $20-25 \%$ of wild tiger populations (Smith, 1993; Kenny et al., 1995).

\section{Discussion}

We have demonstrated in this study that non-invasive photographic sampling is a potentially useful method for estimating densities of tigers in the tropical forests of the Western Forest Complex in Thailand and therefore probably for other similar areas in South-east Asia. Ecological factors, such as climate, topography and present tiger density levels permit the application of this method. The overall probability of capturing a tiger present in the sampled area during the entire survey $\left(\mathrm{M}_{\mathrm{t}+1} / \widehat{N}=0.79\right)$ was $<1$. Therefore, it is critical to use the capture-recapture sampling-based approach (Williams et al., 2002) to deal with the fact that not all tigers present in the study area are likely to be detected.
Based on comparisons of this ad hoc study with earlier surveys in India that employed more rigorous field protocols (Karanth et al., 2002; Nichols \& Karanth, 2002), we recommend the following modifications to future camera trap studies of tigers in the area: (1) The number of camera traps employed in this study was small (10-15). To improve robustness of the statistical inferences of tiger abundance we recommend deployment of at least 40-50 traps, so that the sampled area, the potential number of tiger-exposed traps, and recapture rates can all be increased. (2) The camera trap survey duration should be shorter, preferably $<6$ weeks, to avoid potential violation of population closure assumptions. Furthermore, a pre-designed field survey protocol (Nichols \& Karanth, 2002), which can generate data amenable to straightforward construction of capture histories, should be employed. A larger number of traps would make it easier to implement such a survey design. (3) It would be useful to sample this tiger population photographically on an annual basis to estimate its size and density, as well as other parameters such as longer term rates of survival, recruitment, and permanent and temporary emigration. Robust Design and other recent refinements in capture-recapture analyses (Pollock et al., 1990; Lebreton et al., 1992; Kendall et al., 1997; Williams et al., 2002) facilitate such analyses (Karanth et al., 2006). Reliable monitoring of the responses of tiger population dynamics to threats and conservation interventions can be an effective component of long-term adaptive management.

The observed mean density of $c .4$ tigers per $100 \mathrm{~km}^{2}$ in this study was comparable to the density of 3.3-7.3 tigers per $100 \mathrm{~km}^{2}$ measured in ecologically similar disturbed semi-deciduous forests such as Tadoba, Bhadra, Melghat, Pench and Panna reserves in India (Karanth et al., 2004c). However, better protected Indian reserves that are ecologically comparable to Huai Kha Khaeng, such as Kanha, Bandipur and Nagarahole, support tiger densities that are thrice as high (c. 12 tigers per $100 \mathrm{~km}^{2}$ ). The Huai Kha Khaeng landscape lacks an abundant, social cervid such as the chital Axis axis that accounts for $13-95 \%$ of prey numbers recorded in Indian reserves. However, Eld's deer Cervus eldi, which was extirpated from Huai Kha Khaeng in historical times, is such a species.

Our study area of $477 \mathrm{~km}^{2}$ around Khao Nang Rum research station forms $17 \%$ of the area of Huai Kha Khaeng Wildlife Sanctuary and $2.7 \%$ of the Western Forest Complex. Prima facie, this area appears to support low densities of ungulate prey (Srikosamatara, 1993), and consequently a relatively low density of c. 4 tigers per $100 \mathrm{~km}^{2}$. If the entire landscape surrounding Khao Nang Rum research station supports comparable tiger densities, Huai Kha Khaeng Sanctuary could hold 
113 tigers, and the entire Western Forest Complex c. 720 tigers.

Given the similarity of vegetation types, climate and prey composition between semi-deciduous forests of India and Thailand, their ecological productivities should be comparable. Furthermore, given the similarity in composition of their ungulate prey assemblages, potential maximum prey densities and hence tiger densities should also be similar. Based on tiger density data from well protected Indian reserves (Karanth et al., 2004c), we speculate that Huai Kha Khaeng Sanctuary could potentially hold 338 tigers, and the entire Western Forest Complex $>2,000$ tigers, highlighting the importance of this area for global tiger conservation. Major new conservation initiatives followed on from this study, in particular improved law enforcement under the joint initiatives of the Thailand government and the Wildlife Conservation Society, and we have also implemented an improved camera-trap monitoring system that employs standard closed model photographic capture-recapture sampling of $<60$ days duration (Karanth et al., 2002) using 136 trap sites to sample effectively an area of $1,260 \mathrm{~km}^{2}$.

\section{Acknowledgements}

We are grateful to the Department of National Park, Wildlife, and Plant Conservation, Government of Thailand, for supporting this work. We gratefully acknowledge encouragement received from the following officials of the department: Chatchawan Pisdamkhom, Soontoon Chaiwattana, Kalyanee Boonkerd, Boosabong Kanchanasakha, and Suchitra Changtragoon. We thank the Wildlife Conservation Society, New York, for supporting the involvement of AP, KUK and NSK in this study and for partial funding, and the US Geological Survey's Patuxent Wildlife Research Center for supporting the involvement of JDN. We acknowledge WWFThailand for providing 10 camera traps to start the study. We are grateful to our enthusiastic team of research assistants, Boonyang Srichan, Sompoad Daungsirichantra and Somporn Pakpein for their dedicated fieldwork. We are particularly grateful to James E. Hines for assistance with data analysis.

\section{References}

Burnham, K.P. \& Overton, W.S. (1978) Estimation of the size of a closed population when capture probabilities vary among animals. Biometrika, 65, 625-633.

Carbone, C., Christie, S., Conforti, K., Coulson, T., Franklin, N., Ginsberg, J.R., Griffiths, M., Holden, J., Kawanishi, K., Kinnaird, M., Laidlaw, R., Lynam, A., MacDonald, D.W., Martyr, D., McDougal, C., Nath, L., O'Brien, T., Seidensticker, J., Smith, D., Sunquist, M., Tilson, R. \& Wan Shaharuddin,
W.N. (2001) The use of photographic rates to estimate densities of tigers and other cryptic mammals. Animal Conservation, 4, 75-79.

IUCN (2006) 2006 IUCN Red List of Threatened Species. IUCN, Gland, Switzerland [http:/ / www.redlist.org, accessed 21 August 2007].

Jackson, P. (1993) The status of the tiger in 1993. Cat News, 19, $5-11$.

Karanth, K.U. (1987) Tigers in India: a critical review of field censuses. In Tigers of the World: The Biology, Biopolitics, Management and Conservation of an Endangered Species (eds R.L. Tilson \& U.S. Seal), pp. 118-132. Noyes Publications, Park Ridge, USA.

Karanth, K.U. (1988) Analysis of predator-prey balance in Bandipur Tiger Reserve with reference to census reports. Journal of the Bombay Natural History Society, 85, 1-8.

Karanth, K.U., Chundawat, R.S., Nichols, J.D. \& Kumar, N.S. (2004a) Estimation of tiger densities in the tropical dry forests of Panna, Central India, using photographic capture-recapture sampling. Animal Conservation, 7, 285-290.

Karanth, K.U., Kumar, N.S. \& Nichols, J.D. (2002) Field surveys: estimating absolute densities of tigers using capturerecapture sampling. In Monitoring Tigers and their Prey: A Manual for Researchers, Managers and Conservationists in Tropical Asia (eds K.U. Karanth \& J.D. Nichols), pp. 139-152. Centre for Wildlife Studies, Bangalore, India.

Karanth, K.U. \& Nichols, J.D. (1998) Estimating tiger densities in India from camera trap data using photographic captures and recaptures. Ecology, 79, 2852-2862.

Karanth, K.U., Nichols, J.D. \& Kumar, N.S. (2004b) Photographic sampling of elusive mammals in tropical forests. In Sampling Rare or Elusive Species (ed. W.L. Thompson), pp. 229-247. Island Press, Washington, DC, USA. Karanth, K.U., Nichols, J.D., Kumar, N.S., Link, W.A. \& Hines, J.E. (2004c) Tigers and their prey: predicting carnivore densities from prey abundance. Proceedings of National Academy of Sciences (USA), 101, 4854-4858.

Karanth, K.U., Nichols, J.D., Seidensticker, J., Dinerstein, E., Smith, J.L.D., McDougal, C., Johnsingh, A.J.T., Chundawat, R.S. \& Thapar, V. (2003) Science deficiency in conservation practice: the monitoring of tiger populations in India. Animal Conservation, 6, 141-146.

Karanth, K.U., Nichols, J.D., Kumar, N.S. \& Hines, J.E. (2006) Assessing tiger population dynamics using photographic capture-recapture sampling. Ecology, 87, 2925-2937.

Karanth, K.U. \& Sunquist, M.E. (2000) Behavioural correlates of predation by tiger (Panthera tigris), leopard (Panthera pardus) and dhole (Cuon alpinus) in Nagarahole, India. Journal of Zoology, London, 250, 255-265.

Kawanishi, K. \& Sunquist, M.E. (2004) Conservation status of tigers in a primary rainforest of Peninsular Malaysia. Biological Conservation, 120, 329-344.

Kendall, W.L., Nichols, J.D. \& Hines, J.E. (1997) Estimating temporary emigration and breeding proportions from capture-recapture data with Pollock's Robust Design. Ecology, 78, 563-578

Kenny, J.S., Smith, J.L.D., Starfield, A.M. \& McDougal, C.W. (1995) The long-term effects of tiger poaching on population viability. Conservation Biology, 9, 1127-1133.

Lebreton, J.D., Burnham, K.P., Clobert, J. \& Anderson, D.R. (1992) Modelling survival and testing biological hypotheses using marked animals: a unified approach with case studies. Ecological Monographs, 62, 67-118. 
Nichols, J.D. \& Karanth, K.U. (2002) Statistical concepts: estimating absolute densities of tigers using capturerecapture sampling. In Monitoring Tigers and their Prey: A Manual for Researchers, Managers and Conservationists in Tropical Asia (eds K.U. Karanth \& J.D. Nichols), pp. 121-137. Centre for Wildlife Studies, Bangalore, India.

O'Brien, T.G., Kinnaird, M.F. \& Wibisono, H.T. (2003) Crouching tigers, hidden prey: Sumatran tigers and prey populations in a tropical forest landscape. Animal Conservation, 6, 131-139.

Otis, D.L., Burnham, K.P., White, G.C. \& Anderson, D.R. (1978) Statistical inference from capture data on closed animal populations. Wildlife Monographs, 62, 1-135.

Panwar, H.S. (1980) A note on tiger census technique based on pugmark tracings. Cheetal, 22, 40-46.

Pattanavibool, A. \& Dearden, A. (2002) Fragmentation and wildlife in montane evergreen forest, northern Thailand. Biological Conservation, 107, 155-164.

Petdee, A. (2000) Feeding habits of the tiger (Panthera tigris) in Huai Kha Khaeng Wildlife Sanctuary by fecal analysis. MSc thesis, Faculty of Forestry, Kasetsart University, Bangkok, Thailand.

Pollock, K.H., Nichols, J.D., Brownie, C. \& Hines, J.E. (1990) Statistical inference for capture-recapture experiments. Wildlife Monographs, 107, 1-97.

Rabinowitz, A. (1993) Estimating the Indochinese tiger Panthera tigris corbetti population in Thailand. Biological Conservation, 65, 213-217.

Rabinowitz, A. (1999) The status of the Indochinese tiger: separating fact from fiction. In Riding the Tiger: Tiger Conservation in Human Dominated Landscapes (eds J. Seidensticker, S. Christie \& P. Jackson), pp. 148-165. Cambridge University Press, Cambridge, UK.

Rexstad, E. \& Burnham, K.P. (1991) User's Guide for Interactive Program CAPTURE. Colorado Cooperative Wildlife Research Unit, Colorado State University, Fort Collins, USA.

Seidensticker, J., Christie, S. \& Jackson, P. (1999) Preface. In Riding the Tiger: Tiger Conservation in Human Dominated Landscapes (eds J. Seidensticker, S. Christie \& P. Jackson), pp. xv-xix. Cambridge University Press, Cambridge, UK.

Smith, J.L.D. (1993) The role of dispersal in structuring the Chitwan tiger population. Behaviour, 124, 165-195.

Smith, J.L.D., Tunhikorn, S., Tanhan, S., Simcharoen, S. \& Kanchanasaka, B. (1999) Metapopulation structure of tigers in Thailand. In Riding the Tiger: Tiger Conservation in Human Dominated Landscapes (eds J. Seidensticker, S. Christie \& P. Jackson), pp. 166-175. Cambridge University Press, Cambridge, UK.
Srikosamatara, S. (1993) Density and biomass of large herbivores and other mammals in a dry tropical forest, western Thailand. Journal of Tropical Ecology, 9, 33-43.

Sunquist, M.E. (1981) Social organisation of tigers Panthera tigris in Royal Chitwan National Park, Nepal. Smithsonian Contributions to Zoology, 336, 1-98.

Tunhikorn, S., Smith, J.L.D., Prayurasiddhi, T., Graham, M., Jackson, P. \& Cutter, P. (eds) (2004) Saving Thailand's Tigers: An Action Plan. Ministry of Natural Resources and Environment, Department of National Parks, Wildlife and Plant Conservation, Bangkok, Thailand.

WEFCOM (2004) GIS Database and its Applications for Ecosystem Management. The Western Forest Complex Ecosystem Management Project, Department of National Park, Wildlife, and Plant Conservation, Bangkok, Thailand.

Wegge, P., Pokheral, C.P. \& Jnawali, S.R. (2004) Effects of trapping effort and trap shyness on estimates of tiger abundance from camera trap studies. Animal Conservation, 7, 251-256

White, G.C., Anderson, D.R., Burnham, K.P. \& Otis, D.L. (1982) Capture-Recapture Removal Methods for Sampling Closed Populations. Los Alamos National Laboratory publication no. LA-8787-NERP. Los Alamos, USA.

Williams, B.K., Nichols, J.D. \& Conroy, M.J. (2002) Analysis and Management of Animal Populations. Academic Press, San Diego, USA.

Wilson, K.R. \& Anderson, D.R. (1985) Evaluation of two density estimators of small mammal population size. Journal of Mammalogy, 66, 13-21.

\section{Biographical sketches}

Saksit Simchareon's area of interest is the study of tigers and leopards in the dry forests of Thailand using radio telemetry and camera trapping. He has carried out intensive ecological studies on these species over the past 3 years. Anak Pattanavibool is interested in examining large mammal ecology and conservation issues in Thailand within a landscape ecological framework. K. Ullas Karanth developed camera trap surveys in India with a focus on integrating them with modern animal sampling methods. James D. Nichols works on development of rigorous sampling and analytical methodologies for assessing wildlife populations. N. Samba Kumar specializes in developing field protocols for surveying large mammals in Asian forests. 